\title{
Maximizing co-training opportunities on a traditional health sciences campus
}

\author{
Karen Hughes Miller ${ }^{1}$, Carla P Hermann ${ }^{2}$, Veronnie Faye J ones ${ }^{1}$, Michael Ostapchuk ${ }^{1}$, Pradip D \\ Patel $^{1}$, Michael L Rowland ${ }^{1}$ \\ 1. School of Medicine, University of Louisville, Louisville KY, U.S.A. 2. School of Nursing, University of Louisville, Louisville \\ KY, U.S.A.
}

Correspondence: Karen Hughes Miller. Address: School of Medicine, University of Louisville, 323 East Chestnut St., Abell Administration 511, Louisville KY, U.S.A. Email: Karen.miller@louisville.edu

Received: April 1, 2013

Accepted: May 8, 2013

Online Published: August 8, 2013

DOI : $10.5430 /$ jnep.v3n12p151

URL: http://dx.doi.org/10.5430/jnep.v3n12p151

\begin{abstract}
Both the economics and the science of modern healthcare demand that the best patient care be delivered by an integrated team of healthcare providers, each expert in their own field, but also expert in the ability to function well as a team member. Functioning as a member of a complex team is not intuitive, and even the best educated among us needs additional instruction to do this well. But even the best schools of nursing and medicine, especially those with longer histories and more traditional curricula, may not be designed to support this type of instruction. Practical considerations such as accreditation needs, administration, budget lines, and even physical facilities tend to "silo" instruction by discipline.

We argue that even in institutions with traditional curricula, there are numerous opportunities to co-train nursing, medical, and other healthcare students and faculty if we remain open to possibilities.

This article presents five brief case-studies of co-training events where nursing, medical, and other healthcare students and/or faculty learn in the same environment with minimal administrative effort including: (1) the Certificate in Health Professions Education program; (2) workshops on Increasing Cultural Competence; (3) the iCOPE project in interdisciplinary palliative care; (4) joint daily rounding in an urban children's hospital; and (5) providing care in the Teen Age Parent Program (TAPP).
\end{abstract}

\section{Key words}

Interdisciplinary training, Co-training, Healthcare team training

\section{I ntroduction}

Today's healthcare environment is becoming increasingly reliant on team-based care, cross-trained teams, and interdisciplinary training. Healthcare reform in the US and elsewhere will require today's nursing, medical, and other healthcare students to be able to function well in interdisciplinary teams to maximize efficiency and effectiveness ${ }^{[1]}$. Numerous studies have found that the quality of patient care increased with the increased level of teamwork among healthcare professionals ${ }^{[2-6]}$. And yet, in most schools with a "traditional" curriculum where basic sciences are taught before introducing clinical experiences ${ }^{[7]}$, there is little time and few resources to coordinate formal co-training events. As is 
often the case in post-secondary education, our "silo" style systems of program accreditation, evaluation, and faculty development, work against the idea of co-training the integrated professional teams needed in the real world ${ }^{[2,3,8,9]}$.

Curran and Sharpe ${ }^{[10]}$ approached this problem by developing an interprofessional education curriculum framework - a schematic - that provides healthcare educators and administrators a tool for placing individual learning events into a larger context in order to identify areas where the curriculum is strong and where it is weak. Their work expands that of Barr et al. ${ }^{[11]}$ who identified two types of interdisciplinary training opportunities, "extracurricular" and "crossbar" or formal. Both Curran and Sharpe and Barr et al. encouraged educators and administrators to look at the broad picture including all healthcare disciplines, the progress of learners over time, the need for faculty development to support this effort, and the relevance of the instruction to future practice.

We argue that even in institutions with traditional curricula where educators and administrators may stay within their own discipline by habit, there are numerous opportunities to train nursing, medical, and other healthcare students and faculty together and to increase awareness of the benefits of these events. We simply need to remain alert to the possibilities.

The objective of this article is to present five brief case-studies of co-training events in the University of Louisville (KY) Health Sciences Center where nursing, medical, and other healthcare students and/or faculty learn in the same environment with minimal administrative disruption. These include (1) the Certificate in Health Professions Education program (Michael Rowland, PhD); (2) workshops on Increasing Cultural Competence (V. Faye Jones, MD, PhD); (3) the iCOPE project (Carla Hermann, PhD, RN); (4) joint daily rounding in an urban children's hospital (Pradip Patel, MD); and (5) providing care in the Teen Age Pregnancy Program (TAPP) (Michael Ostapchuk, MD MSEd). Each program has undergone (or is in the process of undergoing) formal instructional program evaluation and we believe they present replicable models that other institutions may consider adopting.

\section{The Certificate in Health Professions Education}

The Certificate in Health Professions Education is a 12-credit hour graduate level program offered through a collaboration of the University of Louisville School of Medicine and the College of Education and Human Development. First offered in 2006, the program is open to all Health Sciences Center (HSC) faculty who wish to earn an academic credential in adult education, program evaluation, and evidence-based research methods. The interdisciplinary instructional teams led by the Course Director include faculty with expertise in clinical medicine and faculty with expertise in adult education. Learners in the program include faculty from nursing, medicine, dentistry, public heath, audiology, and social work ${ }^{[12]}$. The courses are project oriented and learners are encouraged to work in interdisciplinary teams and share their own expertise. Rubrics often include collaboration and/or cooperation as an element of success thus modeling for healthcare faculty how co-training can be introduced as a constructive element in curriculum planning.

The four courses include (1) evidence-based research methods, (2) program and organizational evaluation, (3) teaching in health professions education programs, and (4) adult learning and development. The program has received regional and national attention with the acceptance of a peer reviewed publication ${ }^{[12]}$, formal and informal presentations, and a regional joint university award for instructional development. One of the most sophisticated projects by participants in the program evaluation course resulted in a recently published longitudinal study of instructional outcomes of a pediatric externship ${ }^{[13]}$.

Since 2006, there have been 42 graduates of the program representing all of the health science programs at the University of Louisville. Two graduates (both MDs), applied the graduate credit hours toward completion of their MA in adult education. Enrollment continues to increase and learner satisfaction as measured by formal course evaluations remains high in this constantly evolving program. 


\section{Workshops to increase cultural competence}

As is true in much of the US, the demographic make-up of Kentucky has changed over the past ten years becoming both older and more diverse ${ }^{[14]}$. Although the total population has increased, the white population has decreased thus making Kentucky a "top ten" state for immigrant resettlement ${ }^{[15]}$. This increasing cultural diversity makes it critical to teach learners in every aspect of healthcare how to understand and appreciate the beliefs, knowledge, and ideas of their future patients since these issues have such a strong impact on outcomes ${ }^{[16]}$. Cultural competency is a concept fundamental to the curriculum of all schools on our HSC. Not only does each of our accreditation bodies support interdisciplinary education as a means to enhance culturally competent care ${ }^{[17]}$, but as our states' urban research university, it is an integral part of our mission $^{[18]}$.

Aware of the challenges we were facing in providing culturally competent care ${ }^{[19]}$, we embarked on an ambitious program for interdisciplinary education, and in 2005 piloted cultural competency concepts for first year medical students. Realizing that learners would soon be practicing in interdisciplinary teams, in 2006 we requested participation of nursing, dental and public health students. The goals were (1) to create an emotionally safe learning environment where discussions could occur about cultural beliefs, values, perceptions, and health practices of varying diverse populations; and (2) to teach strategies that promote cultural sensitivity for enhanced healthcare delivery and improved patient outcomes.

We are currently on our seventh year of providing the workshops and have progressed from a faculty-led model to a student-directed model. The workshops are developed and planned by upper-class students from all HSC schools who have gained experience working with a variety of patients and now recognize the importance of culture on healthcare decision making. The students use the qualitative and quantitative data from the previous year to determine speakers and experts to be invited who will assist students in learning about different cultures. Over the past seven years learner evaluations from the workshops have been consistently high indicating learner satisfaction with the variety of information presented, the presenters and the session topics offered. When a topic or an approach falls below expectations, it is reassessed and/or redesigned for improvement the following year.

Among the most popular sessions are discussions of group healthcare beliefs by members of a regional Amish/Mennonite community ${ }^{[20]}$; learning about Islamic health practices; lesbian, gay, bisexual and transgender health issues and concerns; child abuse recognition and prevention; complementary and alternative medicines; substance abuse in the health professions; and health beliefs in the Somali population. Based on what other institutions have found, these co-training workshops, provided early in learners' careers, should provide them with practical information and strategies that will help them throughout their careers ${ }^{[16]}$.

\section{I nterdisciplinary oncology palliative care curriculum}

Cancer is a leading cause of death in the United States ${ }^{[21]}$. Cancer patients have a variety of health needs and palliative care is an integral part of their plan. Although the majority of health professionals in many practice settings will care for patients affected by cancer, health professions students are not always taught palliative care concepts (including interdisciplinary care, an essential component of effective palliative care).

Through a grant funded by the National Cancer Institute, a team of health professionals and educators from nursing, medicine, social work, and chaplaincy, developed and implemented an innovative oncology palliative care curriculum named iCOPE (interdisciplinary curriculum for oncology palliative education). It is open to undergraduate nursing students, medical students, graduate social work students, and clinical pastoral education residents ${ }^{[22]}$.

The curriculum has three primary components: (1) didactic, (2) clinical, and (3) a case management exercise. The didactic component is delivered on-line using case based modules to teach palliative care and interdisciplinary care principles in an interactive format that assists students in application of the content. The clinical portion of the experience places students 
in a care setting focused on palliative care. The length of this experience varies among the disciplines ranging from two to several days. As part of the clinical experience, learners complete a reflective writing exercise that is reviewed by faculty members and then discussed in a group of learners. The third portion of the curriculum is a case management exercise. Learners receive basic information on a patient via a case study. They watch a video of a member of their own discipline performing an assessment on a standardized patient. Using this information, students individually develop a discipline specific plan of care. Following this, students gather in a classroom in interdisciplinary student groups to discuss the patient care plan they created as a student in their particular discipline. Students then watch, as a group, a video of an interdisciplinary patient/family care conference. After viewing the care conference, the student group develops an interdisciplinary care plan and discusses how they would function as an integrated team to provide care to the patient and family.

Although implementation of this project is in the early stages, students from all programs have evaluated their experiences positively. Many challenges exist when educating health professions students together as one group, but the need for interprofessional learning to equip health professions students for future collaborative practice is clear ${ }^{[16,17,19]}$.

\section{J oint rounding}

As healthcare becomes more sophisticated, it has become apparent that no one profession can respond adequately to the complexity of problems that patients present, whether they are children at risk or the growing "baby boom" generation that will survive to an advanced age. Two environments where co-training is evident and critical to excellent patient care in our HSC are the inpatient pediatric ward at Kosair Children's Hospital (KCH, our freestanding children's hospital) and the neonatal intensive care unit at University Hospital (Louisville).

In general Inpatient Pediatric Medicine, the team engages in a style of rounding known as Family Centered Rounds. This is a multidisciplinary approach that includes not only the patient and family, but a team of an attending physician, pediatric and family medicine residents, medical students, nurses and nursing students, and pharmacists and pharmacy students. When needed, other health care providers such as social workers, respiratory therapists, and child life therapists will join the discussion.

After introductions, the medical student or resident who has pre-rounded (gathered overnight information, lab results, and examined the patient) presents the findings to the family and other team members. The resident then discusses the assessment and treatment plan and answers any questions the family may have. Other healthcare providers actively engage in the discussion to determine the plan of care. In this style of medical rounding, the patient and family are placed at the center of the team's discussion and they become actively involved in deciding the best course of action for the patient. The physician team at KCH introduced physician extenders (registered nurse clinicians) in 2011 on the inpatient ward teams. In addition to the benefit of having an inter-professional team, the pediatric residents reduced duty-hour violations and missed core conferences, while increasing their efficiency and proportion of direct patient care ${ }^{[23]}$.

In the Neonatal Intensive Care Unit, an interprofessional team of healthcare professionals and learners can be found participating in rounds in an attempt to draw from all areas of expertise for optimal patient care. Morning rounds consist of an attending physician, neonatal fellow, pediatric and family medicine residents, medical students, a neonatal nurse practitioner and nurse practitioner trainees, a pharmacist and pharmacy students, a nutritionist, and occasionally a social worker. Family members and the nurse for each patient also attend rounds to add any further information needed to help formulate the best treatment plan possible for the baby.

Each baby is seen daily by either a resident or nurse practitioner, who gathers information about the previous 24 hours to present in formal rounds. If the patient has also been assigned to a medical student or nurse practitioner trainee, that learner also gathers the information and examines the patient and will present the patient in formal rounds. 
As reported by Mittal et al., family centered rounds improve parent satisfaction, interpersonal and communication skills, and safety ${ }^{[24]}$. We have observed that the co-training approach allows the attending physician and all other team members (practitioners and learners) to clarify the treatment plan and discuss expected outcomes. It is an educational process for everyone involved including the patient and family, and because of this integration, patient care is made more safe and efficient. Additional investigation of these benefits will take place in the near future, most likely as a resident scholarly study.

\section{Providing care in the Teenage Parent Program (TAPP)}

One of the most popular rotations in the Family Medicine program is working in the Jefferson County Public School (JCPS) Teenage Parent Program (TAPP) where 2nd and 3rd year residents work in teams with attending physicians, nurse practitioners, and nurse practitioner trainees in school based clinics designed specifically for teenaged mothers. This is a very rich service and learning environment because these patients often rely on TAPP for far more than their OB/GYN support. Interdisciplinary teams often deal with patients with asthma, diabetes, obesity, and other chronic care issues in addition to prenatal and postnatal care (including awareness of postpartum depression, a problem more common in adolescent than in adult mothers) ${ }^{[25]}$. In many cases, school based programs provide the only healthcare accessible to these adolescent girls ${ }^{[26]}$. Nurse practitioner trainees, especially, because of the demand for their expertise, play an integral role in dealing with patients with multiple health issues.

The Family Medicine program does not approach the instruction of team based care as unique content but rather integrates it into the program as a whole. Team based care is covered formally and informally in the several days of orientation at the beginning of the program, and reinforced in the first year of residency as family medicine interns rotate through pediatrics including Family Centered Rounds at Kosair Children's Hospital (described in the section above). By the time they rotate through TAPP, they have actively participated in co-training and team based practice, and therefore realize they would quickly be overwhelmed by the workload if they did not rely on other team members.

\section{Conclusion}

Most health professions educators realize intuitively that healthcare students and trainees need multiple instructional events and opportunities to practice interdisciplinary team work and see their respective faculty members working together in a collegial way in order to internalize the importance of mutual respect and reliance among healthcare disciplines. ${ }^{[9]}$ But healthcare education is becoming increasingly more regulated, sometimes with unintended consequences. Strict accreditation requirements, administrative support, and budget lines follow distinct tracks for nursing, medicine, and other disciplines. But rather than fall victim to these limitations, we can stay vigilant for the many day-to-day opportunities to get nursing and medical students together for classroom, lab, and bedside teaching events. By maximizing these natural opportunities for co-training, we can begin to build a culture of collaboration within even traditional institutions and allow learners a preview of their future roles as valued members of healthcare teams rather than as simply a nurse or a physician.

At the University of Louisville, we have seen that even small interdisciplinary projects can have very positive, long-term outcomes as learners and faculty from nursing, medicine, and other healthcare professions begin to look to the other side of the courtyard for new ideas and opportunities. Ideally, this spirit of cooperation will become a habit.

\section{Acknowledgements}

Special thanks to Susan H. Patel, NNP, for her thoughtful contributions.

"In the long history of humankind (and animal kind, too) those who learned to collaborate and improvise most effectively have prevailed” Charles Darwin. 


\section{References}

[1] Tucker K, Wakefield A, Boggis C, Lawson M, Roberts T, Gooch J. Learning together: clinical skills teaching for medical and nursing students. Med Educ. 2003; 37: 630-637. PMid:12834421 http://dx.doi.org/10.1046/j.1365-2923.2003.01558.x

[2] Ferrell BR, Winn R. Medical and nursing education and training opportunities to improve survivorship care. J Clin Oncol. 2006; 24: 5142-5148. PMid:17093277 http://dx.doi.org/10.1200/JCO.2006.06.0970

[3] Headrick LA, Barton AJ, Ogrinc G, Strang C, Aboumatar HJ, Aug MA, et al. Results of an effort to integrate quality and safety into medical and nursing school curricula and foster joint learning. Health Affair. 2012; 31(12): 2669-2680. PMid:23213151 http://dx.doi.org/10.1377/hlthaff.2011.0121

[4] Hobgood C, Sherwood G, Frush K, Hollar D, Maynard L, Foster B, et al. Teamwork training with nursing and medical students: does the method matter Results of an interinstitutional, interdisciplinary collaboration. Qual Saf Health Care.2010; 19(e25). PMid:20427311

[5] Körner M, Ehrhardt H, Steger A-K. Designing an interprofessional training program for shared decision making. J Interpro Care. 2012; http://informahealthcare.com/doi/pdf/10.3109/13561820.2012.711786

[6] Nadolski GJ, Bell M, Brewer BB, Frankel RM, Cushing HE, Brokaw JJ. Evaluating the quality if interaction between medical students and nurses in a large teaching hospital. BMC Med Educ.2006; 6(23). PMid:16638142

[7] Hecker K, Violato C. Medical school curricula: do curricular approaches affect competence in medicine? Fam Med. 2009; 41(6): 420-426. PMid:19492189

[8] Aase I, Aase K, Dieckmann P. Teaching interprofessional teamwork in medical and nursing education in Norway: A content analysis. J Interpro Care. 2012; http://informahealthcare.com/doi/pdf/10.3109/13561820.2012.745489

[9] Hall P, Weaver L. Interdisciplinary education and teamwork: a long and winding road. Med Educ. 2001; 35: 867-875. http://dx.doi.org/10.1046/j.1365-2923.2001.00919.x

[10] Curran V, Sharpe D. A framework for integrating interprofessional education curriculum in the health sciences. Ed F Health. 2007; 20(3).

[11] Barr H, Koppel I, Reeves S, Hammick M, Freeth D. Effective interprofessional education- argument, assumption, and evidence. CAIPE London: united kingdom, Blackwell Publishing; 2005. http://dx.doi.org/10.1002/9780470776445

[12] Miller KH, Greenberg R. Training medical professionals to be educators: Developing a certification in health professions education. JIAMSE. 2007; 17(2): 85-89.

[13] Patel P, Bickel S, Ziegler C, Miller KH. An evaluation of the University of Louisville School of Medicine Pediatric Summer Externship program. Med Sci Ed. 2012; 22(4): 117-127.

[14] Louisville Metro Health Status Report 2012. Louisville Metro Department of Public Health and Wellness. http://www.louisvilleky.gov/NR/rdonlyres/D83AC3D6-0C08-440C-B993-50BB258C573F/0/HealthreportFINAL_12712.pdf

[15] Capp R, Fortuny K, Zimmermann W, Bullock W, Henderson E. A profile of the Foreign-Born in the Louisville Metropolitan Area. The Urban Institute, Metro Louisville Office for International Affairs. http://www.louisvilleky.gov/NR/rdonlyres/CA4C2FD7-E859-482D-9F2B-2800A350F7FC/0/UrbanInstituteFull56PageReport.pdf

[16] Willen S, Carpenter-Song E. Cultural competence in action: lifting the hood on four case studies in medical education. Cult Med Psychiatry.2013; http://link.springer.com/article/10.1007/s11013-013-9319-x

[17] Interprofessional Education Collaborative Expert Panel. Core Competencies for Interprofessional Collaborative Practice: Report of an Expert Panel. Washington, DC: Interprofessional Education Collaborative; 2011.

[18] Mission and Goals, University of Louisville School of Medicine. http://louisville.edu/medschool/about/mission

[19] Pecukonis E, Doyle O, Bliss D, Reducing barriers to interprofessional training: Promoting interprofessional cultural competence. J Interpro Care. 2008; 22(4): 417-428. PMid:18800282 http://dx.doi.org/10.1080/13561820802190442

[20] Belknap B, Wilcox A. Reflecting on Cultural Competency, HEART Humanism in Medicine (9). 2012. https://louisville.edu/medschool/curriculum/heart/heart-newsletter/Volume9final.pdf/at_download/file

[21] American Cancer Society. Cancer Facts \& Figures 2012. Atlanta: American Cancer Society; 2012.

[22] iCOPE program seeks to improve palliative care. University of Louisville Department of Medicine News. http://louisville.edu/medschool/medicine/news/icope-curriculum

[23] Burns C, Multerer S, Stevenson M, Grill J, Boland K, Dahl T, Schwenk K. Extending the overextended resident; a new role for nurse clinicians in the academic pediatric setting. Poster presented at UofL Dept. of Pediatrics 2012 Research Day. http://louisville.edu/medschool/pediatrics/residency/why-choose-uofl/BurnsPoster.pdf/view

[24] Mittal, V, Sigrest T, Ottolini M, Rauch D, Lin H, Landrigan C, Flores G. Family-centered rounds on pediatric wards: a PRIS network survey of US and Canadian hospitalists. Pediatrics. 2010; 126(1): 37-43. PMid:20587682 http://dx.doi.org/10.1542/peds.2009-2364

[25] Phipps MG, Raker CA, Ware CF, Zlotnick, C. Randomized controlled trial to prevent postpartum depression in adolescent mothers. Am J Obstet Gynecol. 2013; 208: 192.e106.

[26] Griswold C, Nasso J, Swinder S, Ellison B, Griswold D, Brooks, M. The prenatal care at school program. J School Nur. 2012. http://jsn.sagepub.com/content/early/2012/11/09/1059840512466111.full.pdf 